\title{
Electrocatalytic oxygen evolution on nickel oxy-hydroxide anodes: Improvement through rejuvenation
}

\author{
Sophia R. Mellsop ${ }^{\mathrm{a}}$, Alister Gardiner ${ }^{\mathrm{b}}$, Aaron T. Marshalla,* \\ ${ }^{a}$ Department of Chemical and Process Engineering, MacDiarmid Institute for Advanced Materials \\ and Nanotechnology, University of Canterbury, Christchurch, New Zealand \\ ${ }^{b}$ Callaghan Innovation, Christchurch, New Zealand
}

\begin{abstract}
The ageing and rejuvenation behaviour of nickel oxy-hydroxide anodes for alkaline water electrolysis is investigated. The anodically formed oxy-hydroxide material is known to age over time causing a decrease in performance. However, this deactivation can be mitigated by temporarily reducing the potential for brief periods. This work looks at continuous rejuvenation of nickel anodes in $30 \mathrm{wt} . \% \mathrm{KOH}$ solution and it is shown that rejuvenation at $0.5 \mathrm{~V}$ vs. $\mathrm{HgHgO}$ for 10 minutes every 100 minutes can prevent ageing of the anode, thus maintaining a low overpotential during galvanostatic oxygen evolution at $50 \mathrm{~mA} \mathrm{~cm}$. It is suggested that the short potentiostatic rejuvenation periods at regular intervals prevents the ratio of $\mathrm{Ni}(\mathrm{IV})$ to $\mathrm{Ni}$ (III) from increasing, thereby maintaining the intrinsic activity of the material. The rejuvenation potential must be above $0.36 \mathrm{~V}$ vs. $\mathrm{HgHgO}$ to ensure it is effective in obtaining good performance (i.e. the material must not reduce to $\mathrm{Ni}(\mathrm{II})$ ). These findings suggest that electrolysis systems using nickel anodes could benefit from direct coupling to fluctuating power sources such as solar or wind, where the variability in their power output could facilitate the rejuvenation of the nickel anode. We estimate that by using the rejuvenation steps, an energy saving of $9 \%$ is possible in an alkaline water electrolyser using nickel anodes.
\end{abstract}

Keywords:

Nickel, Nickel hydroxide, Nickel oxide, Oxygen evolution reaction, Electrocatalysis

${ }^{*}$ Corresponding author

Email address: aaron.marshall@canterbury.ac.nz (Aaron T. Marshall) 


\section{Introduction}

Water electrolysis has long been investigated as a way of producing very pure hydrogen gas from a renewable energy sources. Much of the research has focussed around developing electrocatalysis to improve the efficiency of electrolysers. Important factors to consider when choosing an electrocatalyst include its stability, performance, and cost. Many researchers have investigated nickel oxides as the electrocatalyst for the anodic oxygen evolution reaction [1-7], as it is much less costly than options such as iridium or ruthenium oxide and has reasonably good performance, with a potentials of approximately $750 \mathrm{mV}$ vs. $\mathrm{HgHgO}$ at a current density of $50 \mathrm{~mA} \mathrm{~cm}^{-2}$ [8], and Tafel slopes as low as $38 \mathrm{mV}$. However, the performance stability under anodic conditions can be an issue, resulting in the performance of the electrocatalyst decreasing over time $[6,8]$

As the loss in performance of nickel oxide anodes is generally accepted to be related to structural changes in the oxide, much of the research into nickel oxide electrocatalysts has involved investigating the structure of the electrocatalyst [6, 9-12]. Traditionally four phases are thought to be present as shown in the Bode diagram [13] (Figure 1). The true map of phases and their transformations is likely to be more complex [8] based

on recent research $[8,14-19]$. For example, raman spectroscopy suggests that at the onset of oxygen evolution, $\mathrm{NiOOH}$ transforms to another phase [15] and similarly our cyclic voltammetry work suggested that after extensive oxygen evolution (at least 40 hours at $50 \mathrm{~mA} \mathrm{~cm}^{-2}$ ) an additional phase forms [8]. Many authors have also suggested that over-oxidation causes $\mathrm{Ni}(\mathrm{IV})$ to form [6, 20-23], although there is currently little direct evidence of the formation of $\mathrm{NiO}_{2}$ or other $\mathrm{Ni}(\mathrm{IV})$ species.

Work by Lu and Srinivasan, and Osaka and Yatsuda $[6,7]$ has shown that the activity of a deactivated nickel oxide anode can be recovered by briefly decreasing the 
potential of the anode to values where minimal oxygen evolution occurs ( $1.5 \mathrm{~V}$ vs RHE). This rejuvenation can increase the current density by as much as 3-7 times, compared to that just prior to the rejuvenation process [6]. Lu and Srinivasan [6] also showed that longer rejuvenation times at low potentials, gave larger performance recoveries. One explanation for the ageing or loss in activity (which can be reversed by rejuvenation) is simply that the thickness of the poorly conductive nickel oxide layer increases during the oxygen evolution reaction, thus increasing the ohmic resistance of the electrode. However the work of Lu and Srinivasan appears to rule this out as the main cause, and instead it has been suggested that the activity is improved by a thin layer of more highly active electrocatalyst (perhaps $\beta$-NiOOH) forming on the surface of the oxide during rejuvenation, and it is this which controls the specific activity, rather than the structure of the bulk oxide [6].

Here the rejuvenation of a nickel oxide electrode is investigated as a way of improving the activity for the oxygen evolution reaction. Specifically, the role of rejuvenation

potential, time and frequency is examined, as well as the implications of the ageing and rejuvenation of nickel oxide anodes on the measurement of steady-state polarisation data.

\section{Experimental}

Nickel foil electrodes (Sigma-Aldrich $\geq 99.9 \%$ ), with a surface area of $1 \mathrm{~cm}^{2}$, were cleaned in acetone for 5 minutes, ultrasonicated in water, etched in $1 \mathrm{M}$ hydrochloric acid solution with $5.25 \mathrm{~g} \mathrm{~L}^{-1}$ of hydrogen peroxide for 15 minutes, then finally rinsed in deionised water. Contact to the nickel foil was achieved by spot welding a nickel wire (0.5 mm diameter) to a corner of the foil. This wire was insulated from the electrolyte using an inert thermosetting polymer. 
A Gamry Instruments Reference 3000 potentiostat was used for electrochemical analysis. The electrochemical tests were performed in $30 \mathrm{wt} \% \mathrm{KOH}$ solution in a thermostatically controlled PTFE cell at $25^{\circ} \mathrm{C}$. Nickel foil $\left(30 \mathrm{~cm}^{2}\right)$ was used as the counter electrode along with a $\mathrm{Hg} / \mathrm{HgO}(\mathrm{KOH})$ reference electrode. All potentials are referenced to the $\mathrm{HgHgO}(30 \% \mathrm{KOH})$. Unless stated otherwise the following pretreatment procedure was used on the nickel electrodes: the freshly etched nickel electrodes were subjected to potentiostatic electrochemical impedance spectroscopy (EIS) at $0 \mathrm{~V}$ (to obtain the ohmic resistance between the reference and working electrodes) over the frequency range $0.2-100,000 \mathrm{~Hz}$ at $5 \mathrm{mV}$ rms before the potential was held at $-100 \mathrm{mV}$ for 2 hours (to ensure the electrode surface was covered in the $\alpha-\mathrm{Ni}(\mathrm{OH})_{2}$ phase), then cycled at $50 \mathrm{mV} \mathrm{s}^{-1}$ for 5 to 20 cycles between $-0.1 \mathrm{~V}$ and $0.65 \mathrm{~V}$.

All rejuvenation measurements involved galvanostatic oxygen evolution at $50 \mathrm{~mA} \mathrm{~cm} \mathrm{~cm}^{-2}$ coupled with potentiostatic rejuvenation periods at open circuit potential (OCP), $0.5 \mathrm{~V}$, or $0.2 \mathrm{~V}$. Cyclic voltammetry was performed at the end of all oxygen evolution/rejuvenation measurements at $50 \mathrm{mV} \mathrm{s}^{-1}$ from $-0.1 \mathrm{~V}$ to $0.65 \mathrm{~V}$. Slow linear scanning voltammograms discussed in Section were performed at a rate of $0.2 \mathrm{mV} \mathrm{s}^{-1}$ following the initial electrode pretreatment and 40-80 hours of galvanostatic oxygen evolution at $50 \mathrm{~mA} \mathrm{~cm}$. All potential measurements were were corrected for the uncompensated IR drop post measurement using the value determined by EIS.

\section{Results and Discussion}

\subsection{Galvanostatic Ageing and Rejuvenation}

When a nickel electrode is subjected to continuous potential cycling (e.g. cyclic

voltammetry), a change in the oxide structure is known to occur $[1,8,24]$. Here the cyclic voltammogram after 200 cycles is noticeably different to that after only 5 cycles 
(Figure 2). It is well known that this cycling causes a transition from the $\alpha-\mathrm{Ni}(\mathrm{OH})_{2}$ $/ \gamma$-NiOOH solid-state redox couple to the $\beta$-Ni(OH $)_{2} / \beta$-NiOOH couple $[1,8,24]$. During cycling, the peak labelled A1, decreases in size while A1' and A2 grow. A2 stops growing at 200 cycles while A1' continues to grow. While it is unclear whether A1' or A2 corresponds to the oxidation of $\beta-\mathrm{Ni}(\mathrm{OH})_{2}$ to $\beta-\mathrm{NiOOH}$, galvanostatic oxygen evolution after cycling (data not shown) reveals that at least one of these peaks corresponds to the formation of a more active phase compared to the $\gamma$-NiOOH phase (which forms from $\alpha-\mathrm{Ni}(\mathrm{OH})_{2}$, peak A1). This is consistent with the recent work which clearly shows that potential cycling can improve the activity of a nickel oxide electrode towards the oxygen evolution reaction [2].

For an electrode which has only undergone 5-20 cycles during the initial electrode pretreatment (and thus is only in the $\alpha-\mathrm{Ni}(\mathrm{OH})_{2} / \gamma-\mathrm{NiOOH}$ structure), a very characteristic ageing behaviour is observed during galvanostatic oxygen evolution (Figure 3a). The galvanostatic ageing can be split into five periods: an initial decrease in overpotential (period 1), a period of good performance (period 2), a slow increase in overpotential (period 3), a steep increase in overpotential (period 4), and finally a stable high potential (period 5). While it is clear that initially only the $\alpha-\gamma$ phases are present at the start of galvanostatic ageing, once period 4 is reached, a second pair of peaks appear in the voltammograms (A3 and C3) (Figure 3b). By comparing the voltammogram from this galvanostatically aged $\alpha-\mathrm{Ni}(\mathrm{OH})_{2} / \gamma$-NiOOH anode with that in Figure 2 , it is clear that peaks A3 and C3 arise from a different solid-state redox pair to those found after ageing by cyclic voltammetry. We suggest that peaks A3 and C3 result from the formation of a structure containing $\mathrm{Ni}(\mathrm{IV})$ [8].

While nickel oxide anodes (initially with the $\alpha / \gamma$ structure) are slowly deactivated during galvanostatic oxygen evolution, it is observed that the activity can be partially 
recovered if the potential is temporary decreased to $0.5 \mathrm{~V}$ before return back to the galvanostatic oxygen evolution (Figure 4). An example of this is given in Figure 4, where oxygen evolution at $50 \mathrm{~mA} \mathrm{~cm}^{-2}$ is interrupted after 40 hours by a 10 minute rejuvenation step at $0.5 \mathrm{~V}$. Just prior the the rejuvenation step, the anode potential is $0.82 \mathrm{~V}$ (and increasing), whereas immediately after the rejuvenation step the potential is about $0.8 \mathrm{~V}$ (and approximately stable for 5-10 hours), i.e. a rejuvenation of $20 \mathrm{mV}$. This rejuvenation affect is also seen in other work $[6,7]$ and has been suggested to be related to a rapid change in the surface of the oxide, upon decreasing the potential, to a form which is more intrinsically active toward the oxygen evolution reaction [6]. This active oxide layer is said to have a higher ratio of $\mathrm{Ni}(\mathrm{III})$ to $\mathrm{Ni}(\mathrm{IV})$ than the layer before rejuvenation, and is consistent with the Pourbaix diagram for nickel [25] which shows that approximately $0.6 \mathrm{~V}$ vs $\mathrm{HgHgO}$ is the transition potential for $\mathrm{Ni}(\mathrm{III})$ to $\mathrm{Ni}(\mathrm{IV})$.

After the brief period of stable anode potential after the rejuvenation step, the potential increases again and follows the characteristic deactivation or ageing process (in

other words, the rejuvenation is only temporary). However, it takes approximately 15 hours of galvanostatic oxygen evolution (post rejuvenation) before the anode potential reaches the same value as just prior to rejuvenation, despite the rather brief duration (10 minutes) of the rejuvenation step. Similar results are described by Lu and Srinivasan [6] but under potentiostatic oxygen evolution conditions.

\subsection{Periodic or continuous rejuvenation}

As brief rejuvenation steps appear to improve the performance for many hours, it was hypothesized that by carrying out the rejuvenation steps periodically throughout oxygen evolution, the slow ageing and loss in performance found for nickel oxide anodes may be prevented. Thus continuous rejuvenation was carried out by periodically holding the potential of the anode at $0.5 \mathrm{~V}$ for 10 minutes every 100 minutes during 
oxygen evolution at $50 \mathrm{~mA} \mathrm{~cm}^{-2}$ (Figure 5a). By rejuvenating the anode periodically, the potential (at $50 \mathrm{~mA} \mathrm{~cm}^{-2}$ ) can maintained at low values for at least 120 hours (compare Figure 3a with Figure 5a). We note that the average anode potential over the 120 hours is very similar to the minimum potential found during a normal long-term oxygen evolution measurement at $50 \mathrm{~mA} \mathrm{~cm}^{-2}$. Furthermore, unlike the normal galvanostatic operation, there is very little change in the cyclic voltammograms before and after the 120 hours of oxygen evolution under the periodic rejuvenation operation. We therefore conclude that the periodic rejuvenation steps has prevented the slow phase transformation which normally occurs during long-term oxygen evolution. A similar periodic rejuvenation operation was also attempted wherein the rejuvenation step was reduced to 1 minute at $0.5 \mathrm{~V}$ every 100 minutes. Under this mode of operation, no significant improvement over the normal galvanostatic operation was observed (i.e. the rejuvenation time was insufficient), and thus further measurements are required to optimise the rejuvenation time.

The effect of rejuvenation potential was also examined by conducting periodic rejuvenation operation using a lower potential of $0.2 \mathrm{~V}$ (Figure 6a). This potential was selected to be sufficiently cathodic to ensure that the oxide undergoes the $\mathrm{NiOOH}$ to $\mathrm{Ni}(\mathrm{OH})_{2}$ phase change during the rejuvenation step. This clearly gives significantly different results than those seen for a rejuvenation potential of $0.5 \mathrm{~V}$. Overall, the activity is not as good as that achievable when the rejuvenation potential is $0.5 \mathrm{~V}$, nor does the potential reach the same minimum as when no rejuvenation is performed. However, as with the rejuvenation potential of $0.5 \mathrm{~V}$, the severe deactivation (i.e. when the potential slowly increases to $1-1.05 \mathrm{~V}$ ) which normally occurs under galvanostatic operation, is not observed.

Examining the voltammogram measured after the 120 hours of periodic rejuvena- 
tion, immediately reveals that changes to the oxide structure has occurred over the 120 hours of operation (Figure 6b). A slight anodic shift in the potentials of peaks A1 and C1 have occurred and a large anodic peak at approximately $0.48 \mathrm{~V}$ is observed, which we attribute to the process identified by peak A2 in Figure 2. As both A1 and C1 have shifted to higher potentials, it is unlikely that this shift is due to the transition of the oxide layer towards the $\beta / \beta$ structure as the cathodic peak corresponding to the reduction of $\beta-\mathrm{NiOOH}$ to $\beta-\mathrm{Ni}(\mathrm{OH})_{2}$ is normally at a more cathodic potential than the $\gamma-\mathrm{NiOOH}$ to $\alpha-\mathrm{Ni}(\mathrm{OH})_{2}$ reaction $[8,10,16]$. One could suggest that these two peaks equivalent to those labelled as A3 and C3 in Figure 3b, but this is also unlikely as A3 and C3 are usually observed along with A1 and C1 [8, 22]. One may also argue that this shift is caused by a shift in the potential of the reference electrode, however this is unlikely due to the regular testing of reference electrodes against stable standards in our laboratory. Despite the uncertainty in the origin of this peak shift, what is more important is the significant growth in peak A2 and the implications that this peak has on the anodes activity towards the oxygen evolution reaction. Given that most authors refer to $\beta$-NiOOH as the "right type of oxide" for oxygen evolution [6], the fact that A2 dominates the voltammogram and the performance is relatively poor suggests A2 is not associated with the $\beta$ - $\beta$ reaction. However, given the uncertainty in the literature regarding identification of the peaks on a nickel oxide voltammogram, a more accurate statement is possibly that the phase which forms during peak A2 is not as active as the phase which forms during peak A1'.

While periodic rejuvenation could enable water electrolysis systems to operate more efficiently, incorporating potentiostatic rejuvenation steps within an electrolyser's control system may add an unacceptable degree of complexity. An alternative and more simple approach would be to use periodic open circuit rejuvenation steps throughout 
galvanostatic operation. Typically, for this type of anode, the open circuit potential (OCP) is around $0.3-0.5 \mathrm{~V}$ (i.e. similar to the potential which does indeed rejuvenate the anode). For this reason the long-term behaviour of the nickel oxide anode was investigated at $50 \mathrm{~mA} \mathrm{~cm}^{-2}$ with periodic rejuvenation steps at OCP for 10 minutes every 100 minutes (Figure 7a). Given the importance which the rejuvenation potential has on the overall long-term behaviour of the anode, OCP during the 10 minute rejuvenation segments was also measured (Figure 7a). During the first 10 hours the potential at $50 \mathrm{~mA} \mathrm{~cm}^{-2}$ is similar to when a $0.2 \mathrm{~V}$ rejuvenation step was used (Figure 6), although after this the activity of the anode improves to the point where it is almost equal to that achieved by the electrode operating with the $0.5 \mathrm{~V}$ rejuvenation steps (Figure 5a). At the point where there is a noticeable improvement in the activity (after approximately 10 hours - vertical line on Figure 7), the average OCP measured during the open circuit rejuvenation steps increases above $0.36 \mathrm{~V}$. This potential is approximately equal to the potential at which the cathodic reduction peak starts in the cyclic voltammogram (Figure 7). From this, it is inferred that in order for rejuvenation to take place, and enable the activity of the anode to remain high, the rejuvenation potential must stay above $0.36 \mathrm{~V}$, i.e. the nickel oxide must not be reduced below $\mathrm{Ni}(\mathrm{III})$. The cyclic voltammogram measured after the 120 hours is similar to that measured prior to the long-term operation (albeit with slightly larger peaks). This would be expected as the this operation enabled the anode to operate at $50 \mathrm{~mA} \mathrm{~cm}^{-2}$ with similar anode potentials to the electrode with rejuvenation steps controlled at $0.5 \mathrm{~V}$.

While the successful rejuvenation appears to require brief periods at potentials between 0.36-0.5 V, the mechanism is not completely clear. Lu and Srinivasan suggest that it is the reduction of $\mathrm{Ni}(\mathrm{IV})$ (formed during oxygen evolution) to $\mathrm{Ni}(\mathrm{III})$ which is responsible for the rejuvenation [6], however our attempts to measure the charge or cur- 
rent associated with this hypothesised reduction process were unsuccessful due to the presence of anodic oxygen evolution which occurs above approximately $0.3 \mathrm{~V}$. In-situ x-ray absorption spectroscopy was also unsuccessful in identifying any oxide structures containing $\mathrm{Ni}(\mathrm{IV})$ during oxygen evolution (up to $0.665 \mathrm{~V}$ ) [8], although it is important to note that these in-situ measurements were performed on $\mathrm{Ni}$ anodes which had not undergone extensive galvanostatic ageing. Thus it is likely that the concentration of any $\mathrm{Ni}(\mathrm{IV})$ species (which may be responsible for the deactivation) present in the oxide film will very low and undetectable by these XAS measurements. However others have measured XAS spectra consistent with the $\mathrm{Ni}$ oxide films containing $\mathrm{Ni}(\mathrm{IV})$ species after extensive anodic polarisation [26].

\subsection{Linear Scanning Voltammetry and Polarisation Curves}

The characteristic ageing of a nickel oxide anode (initially with the $\alpha$ - $\gamma$ oxide structure) clearly complicates the measurement of a steady-state polarisation curve. To illustrate this, polarisation curves were measured after 40 and 80 hours of galvanostatic oxygen evolution at $50 \mathrm{~mA} \mathrm{~cm}^{-2}$ (Figure 8), and as expected the activity of the anode which had undergone oxygen evolution for only 40 hours was more active than that after 80 hours of oxygen evolution. What is more problematic for the accurate determination of the electrodes activity, is that the potential at $50 \mathrm{~mA} \mathrm{~cm} \mathrm{~cm}^{-2}$ just prior to the measurement of these polarisation curves was considerable different to that measured during the polarisation curve (Figure 8). This is easily explained by the rejuvenation effect (e.g. Figure 4), as the polarisation curve was obtained by stepping the potential from that at $50 \mathrm{~mA} \mathrm{~cm}^{-2}(0.86 \mathrm{~V}$ after 40 hours or $1.07 \mathrm{~V}$ after 80 hours $)$ to $0.5 \mathrm{~V}$ before increasing the potential at $0.2 \mathrm{mV} \mathrm{s}^{-1}$. It is also observed that the polarisation curve for the anode after 40 hours of OER, has significant hysteresis between the forward and reverse sweep directions. 
To better understand the hysteresis between the forward and reverse sweeps, three sweeps were performed in succession (reverse, forward, reverse) immediately after galvanostatic OER at $50 \mathrm{~mA} \mathrm{~cm}^{-2}$ for 45 hours (Figure 9). The use of a reverse sweep from the OER potential at $50 \mathrm{~mA} \mathrm{~cm}^{-2}$ ensures that the rejuvenation effect cannot affect the shape of the polarisation curve until the potential reaches a sufficiently low potential (e.g. $0.5 \mathrm{~V}$ ). The first reverse sweep starts at approx $0.82 \mathrm{~V}$ (close to $50 \mathrm{~mA} \mathrm{~cm}{ }^{-2}$ ) and has a steeper slope than the subsequent two curves. On the following forward sweep the slope has decreased and the performance has improved i.e., during the small amount of time at low potentials (e.g. $0.5 \mathrm{~V}$ ) the material has rejuvenated and the performance improved. During the second reverse sweep (Reverse 2) the performance decreases again but not enough for the potential to follow the same path as that seen for "Reverse 1", suggesting that the rejuvenation process is noticeably faster than the ageing process - consistent with our findings that short rejuvenation steps can result in long periods of rejuvenated performance (section 3.2).

\section{Implications of rejuvenation in water electrolysis systems}

The potentials shown in the Figures 3 and 5 were used to calculate the approximate energy requirements for a simple single cell electrolysis cell. A constant potential

for the hydrogen evolution reaction of $-1.17 \mathrm{~V}$ vs $\mathrm{HgHgO}$ [27] at $50 \mathrm{~mA} \mathrm{~cm}^{-2}$ and a negligible ohmic loss were assumed. We have also assumed that the rejuvenation steps will have little influence on the stability and performance of the cathodic hydrogen evolution reaction, as the cathode is unlikely change from its metallic state during the brief rejuvenation steps. For the case of no rejuvenation, we have used an anode potential of $0.918 \mathrm{~V}$ vs $\mathrm{HgHgO}$ (the average potential from Figure 3), although we note that for long term operation the likely anode potential will be even higher at around 1.0 $\mathrm{V}$ vs $\mathrm{HgHgO}$. For an anode operating with rejuvenation, the average measured 
anode potential at $50 \mathrm{~mA} \mathrm{~cm}^{-2}$ is only $0.73 \mathrm{~V}$ vs $\mathrm{HgHgO}$ (Figure 5a). Thus under these conditions, the estimated cell potential for a galvanostatic electrolyser would be $0.918+1.17=2.088 \mathrm{~V}$, whereas the electrolyser with rejuvenation would only require approximately $0.747+1.17=1.917 \mathrm{~V}$. Thus at $50 \mathrm{~mA} \mathrm{~cm}^{-2}$, to supply the charge required to generate $1 \mathrm{~kg} \mathrm{H}$ gas $\left(9.6485 \times 10^{3} \mathrm{C}\right)$ a galvanostatic electrolyser would require a total of $56 \mathrm{kWhr}\left(4.57 \mathrm{kWh}\right.$ per normal $\left.\mathrm{m}^{3} \mathrm{H}_{2}\right)$ while a cell with rejuvenation requires only $51 \mathrm{kWhr}\left(4.16 \mathrm{kWh}\right.$ per normal $\left.\mathrm{m}^{3} \mathrm{H}_{2}\right)$. This equates to an energy saving of approximately $9 \%$. We do note that due to the minimal electrolysis during the rejuvenation periods, either larger electrodes or longer times would be required to produce a given quantity of $\mathrm{H}_{2}$, but complete analysis of the economic aspects of these two operation modes is beyond the scope of this paper.

\section{Conclusions}

Electrocatalytic nickel oxide anodes slowly age during the oxygen evolution reaction in alkaline electrolytes. When the performance starts to decrease significantly, a clear change in the anodes cyclic voltammetry response is observed, indicating a change in the anode's surface chemistry. This ageing can be prevented through a rejuvenation process.

Holding the potential at $0.5 \mathrm{~V}$ vs $\mathrm{HgHgO}$ for 10 minutes every 100 minutes of galvanostatic oxygen evolution has been shown to rejuvenate the material and prevent the decrease in performance due to anode ageing. It is thought that this rejuvenation prevents an increase in the ratio of $\mathrm{Ni}(\mathrm{IV})$ to $\mathrm{Ni}(\mathrm{III})$. We have also shown that the rejuvenation potential can be lower than $0.5 \mathrm{~V}$ but it must not allow the surface to reduce to $\mathrm{Ni}(\mathrm{II})$ (i.e. the rejuvenation potential must be above $0.36 \mathrm{~V}$ ). Through rejuvenating the nickel anode material at regular intervals, hydrogen can be produced with $9 \%$ less energy compared to an electrolyser running under continuous galvanostatic operation. 


\section{Acknowledgements}

Funding for this work was provided under MSI Contract C08X1002.

\section{References}

[1] M. Lyons, M. Brandon, The Oxygen Evolution Reaction on Passive Oxide Covered Transition Metal Electrodes in Aqueous Alkaline Solution . Part I-Nickel, International Journal of Electrochemical Science 3 (12) (2008) 1386-1424.

[2] I. Godwin, M. Lyons, Enhanced Oxygen Evolution at Hydrous Nickel Oxide Electrodes via Electrochemical Aging in Alkaline Solution, Electrochemistry Communications 32 (2013) 39-42.

[3] M. Vukovi, Voltammetry and anodic stability of a hydrous oxide film on a nickel electrode in alkaline solution, Journal of Applied Electrochemistry 24 (1994) 878882.

[4] M. Cappadonia, J. Divisek, T. von der Heyden, U. Stimming, Oxygen evolution at nickel anodes in concentrated alkaline solution, Electrochimica Acta 39 (1112) (1994) 1559-1564.

[5] Y. Huang, C. Lai, P. Wu, L. Chen, Ni Inverse Opals for Water Electrolysis in an Alkaline Electrolyte, Journal of The Electrochemical Society 157 (3) (2010) P18.

[6] P. Lu, S. Srinivasan, Electrochemical-Ellipsometric Studies of Oxide Film Formed on Nickel during Oxygen Evolution, Journal of The Electrochemical Society 125 (9) (1978) 1416-1422. 
[7] T. Osaka, Y. Yatsuda, A study on time-dependence of the oxygen evolution reaction on nickel by FFT impedance measurement, Electrochimica Acta 29 (5) (1984) 677-681.

[8] S. Mellsop, A. Gardiner, B. Johannessen, A. Marshall, Structure and transformation of oxy-hydroxide films on $\mathrm{Ni}$ anodes below and above the oxygen evolution potential in alkaline electrolytes, Electrochimica Acta 168 (2015) 356-364.

[9] R. Barnard, C. Randell, Studies concerning charged nickel hydroxide electrodes, Journal of Power Sources 9 (2) (1983) 185-204.

[10] M. Lyons, A. Cakara, P. Brien, I. Godwin, R. Doyle, Redox , pH sensing and Electrolytic Water Splitting Properties of Electrochemically Generated Nickel Hydroxide Thin Films in Aqueous Alkaline Solution, International Journal of Electrochemical Science 7 (12) (2012) 11768-11795.

[11] D. Hall, D. Lockwood, S. Poirier, C. Bock, B. MacDougall, Raman and infrared spectroscopy of $\alpha$ and $\beta$ phases of thin nickel hydroxide films electrochemically formed on nickel., The journal of physical chemistry. A 116 (25) (2012) 6771-6784.

[12] S. Medway, C. Lucas, A. Kowal, R. Nichols, D. Johnson, In situ studies of the oxidation of nickel electrodes in alkaline solution, Journal of Electroanalytical Chemistry 587 (1) (2006) 172-181.

[13] H. Bode, K. Dehmelt, J. Witte, Zur kenntnis der nickelhydroxidelektrodeI.Über das nickel (II)-hydroxidhydrat, Electrochimica Acta 11 (8) (1966) 1079-1087.

[14] T. Ohligschlager, G. Schwitzgebel, EQCM contributions to the reactions of the nickel oxide electrode, Physical Chemistry Chemical Physics 3 (23) (2001) 52905296. 
[15] B. Yeo, A. Bell, In Situ Raman Study of Nickel Oxide and Gold-Supported Nickel Oxide Catalysts for the Electrochemical Evolution of Oxygen, The Journal of Physical Chemistry C 116 (15) (2012) 8394-8400.

[16] M. Lyons, R. Doyle, I. Godwin, M. O’Brien, L. Russell, Hydrous Nickel Oxide: Redox Switching and the Oxygen Evolution Reaction in Aqueous Alkaline Solution, Journal of the Electrochemical Society 159 (12) (2012) H932-H944.

[17] M. Lyons, L. Russell, M. Brien, R. Doyle, I. Godwin, M. Brandon, Redox Switching and Oxygen Evolution at Hydrous Oxyhydroxide Modified Nickel Electrodes in Aqueous Alkaline Solution : Effect of Hydrous Oxide Thickness and Base Concentration, International Journal of Electrochemical Science 7 (4) (2012) 2710-2763.

[18] L. Burke, T. Twomey, Voltammetric behaviour of nickel in base with particular reference to thick oxide growth, Journal of Electroanalytical Chemistry and Interfacial Electrochemistry 162 (1-2) (1984) 101-119.

[19] L. de Souza, F. Kong, F. McLarnon, R. Muller, Spectroscopic ellipsometry study of nickel oxidation in alkaline solution, Electrochimica Acta 42 (8) (1997) 1253-1267.

[20] G. Briggs, W. Wynne-Jones, The nickel oxide electrode. Part 3, Transactions of the Faraday Society 52 (1956) 1272-1281.

[21] E. Jones, W. Wynne-Jones, The Nickel Oxide Electrode. Part 2, Transactions of the Faraday Society 52 (1956) 1260-1272.

[22] L. Liu, Z. Zhou, C. Peng, Sonochemical intercalation synthesis of nano $\gamma$-nickel oxyhydroxide: Structure and electrochemical properties, Electrochimica Acta 54 (2) (2008) 434-441. 
[23] D. Davies, W. Barker, Influence of $\mathrm{pH}$ on Corrosion and Passivation of Nickel, Corrosion 20 (1964) 47t.

[24] I. Casella, M. Guascito, M. Sannazzaro, Voltammetric and XPS investigations of nickel hydroxide electrochemically dispersed on gold surface electrodes, Journal of Electroanalytical Chemistry 462 (2) (1999) 202-210.

[25] M. Pourbaix, Atlas of Electrochemical Equilibria in Aquesous Solutions, Pergamon Press, New York, 1966.

[26] W. E. O’Grady, K. I. Pandya, K. E. Swider, D. A. Corrigan, In Situ X-Ray Absorption Near Edge Structure Evidence for Quadrivalent Nickel in Nickel Battery Electrodes, Journal of the Electrochemical Society 143 (5) (1996) 1613-1617.

[27] L. Vázquez-Gómez, S. Cattarin, P. Guerriero, M. Musiani, Hydrogen evolution on porous $\mathrm{Ni}$ cathodes modified by spontaneous deposition of Ru or Ir, Electrochimica Acta 53 (28) (2008) 8310-8318. 


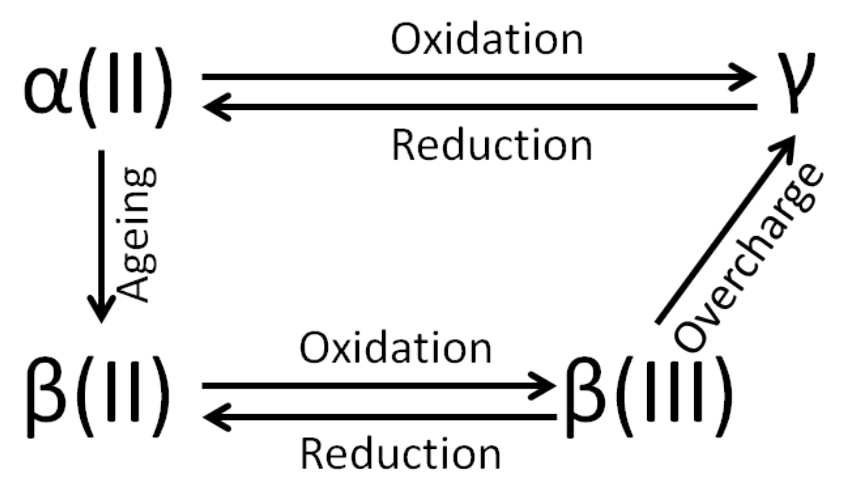

Figure 1: Bode diagram of known nickel phases.

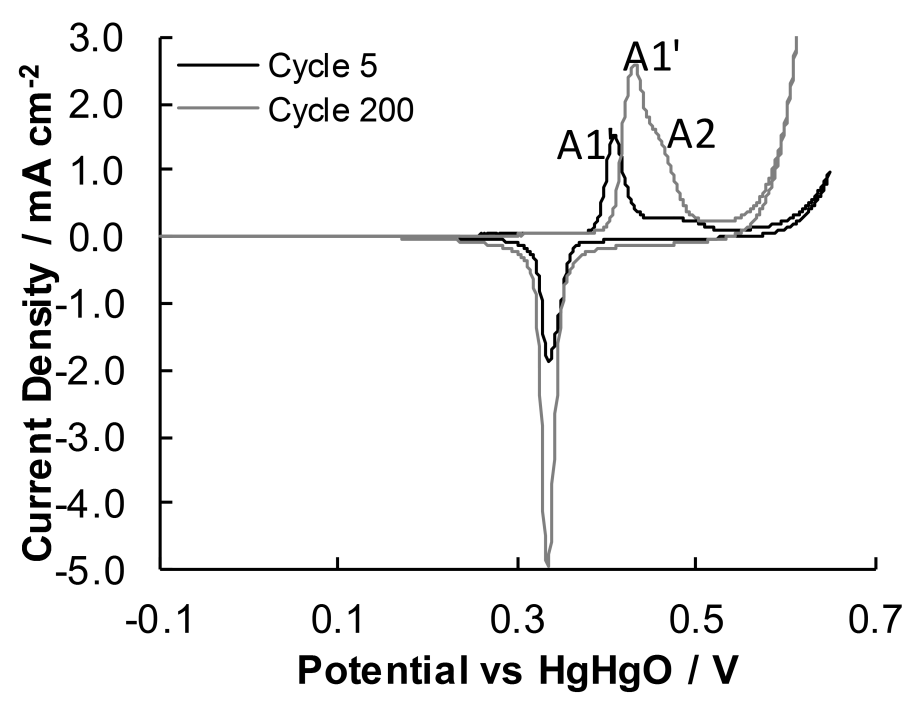

Figure 2: Cycle 5 and 200 of cyclic voltammograms performed at $50 \mathrm{mV} \mathrm{s}^{-1}$ between $-0.1 \mathrm{~V}$ and $0.65 \mathrm{~V}$ following a pretreatment of potentiostatic EIS at $0 \mathrm{~V}$ over the frequency range $0.2-100,000 \mathrm{~Hz}$ at $5 \mathrm{mV} \mathrm{rms}$ and constant potential at $-100 \mathrm{mV}$ for 2 hours. 

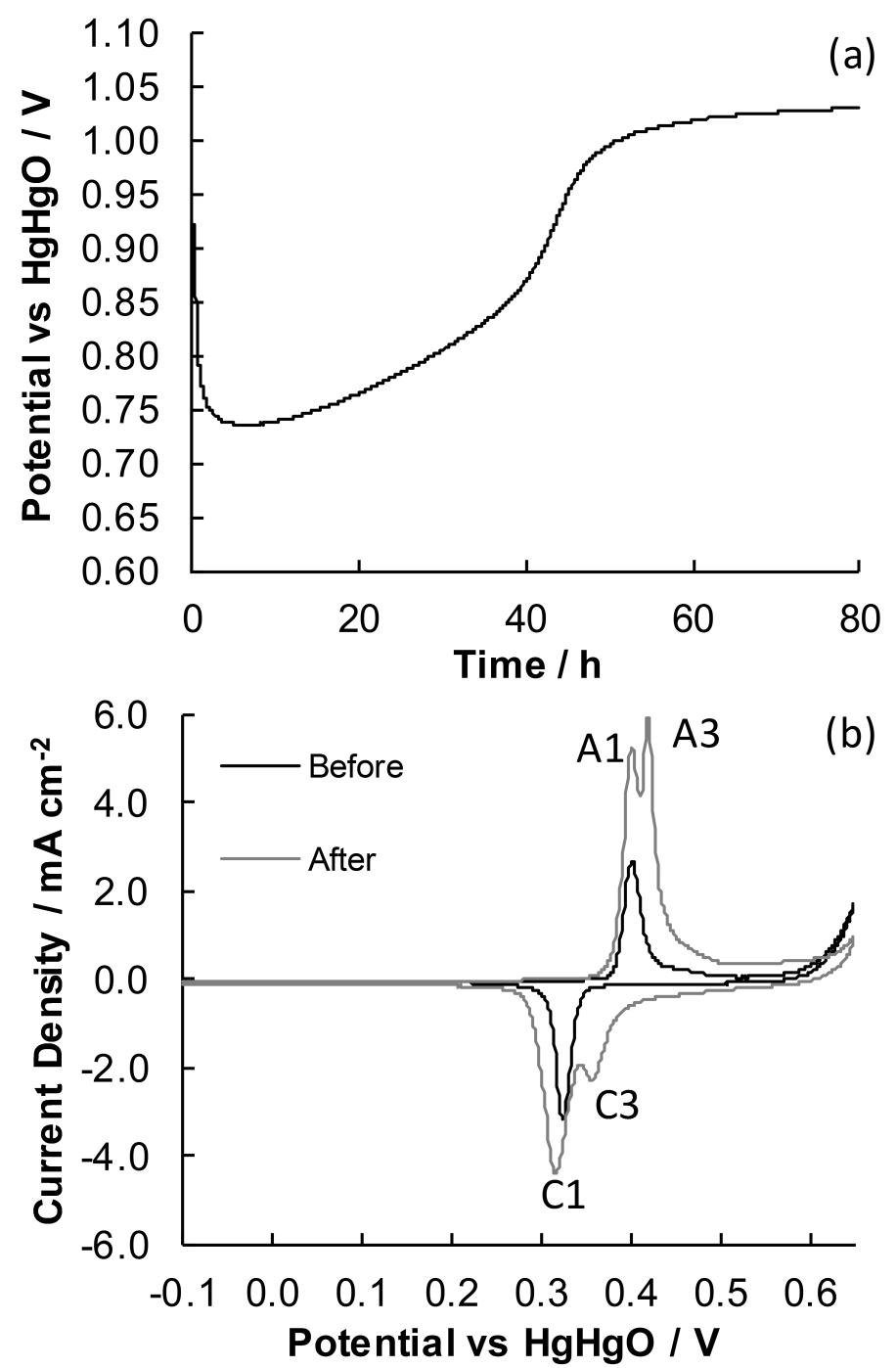

Figure 3: Anode potential over 80 hours of galvanostatic oxygen evolution at $50 \mathrm{~mA} \mathrm{~cm}{ }^{-2}$ (a) and cyclic voltammograms performed before and after the galvanostatic period (b). 


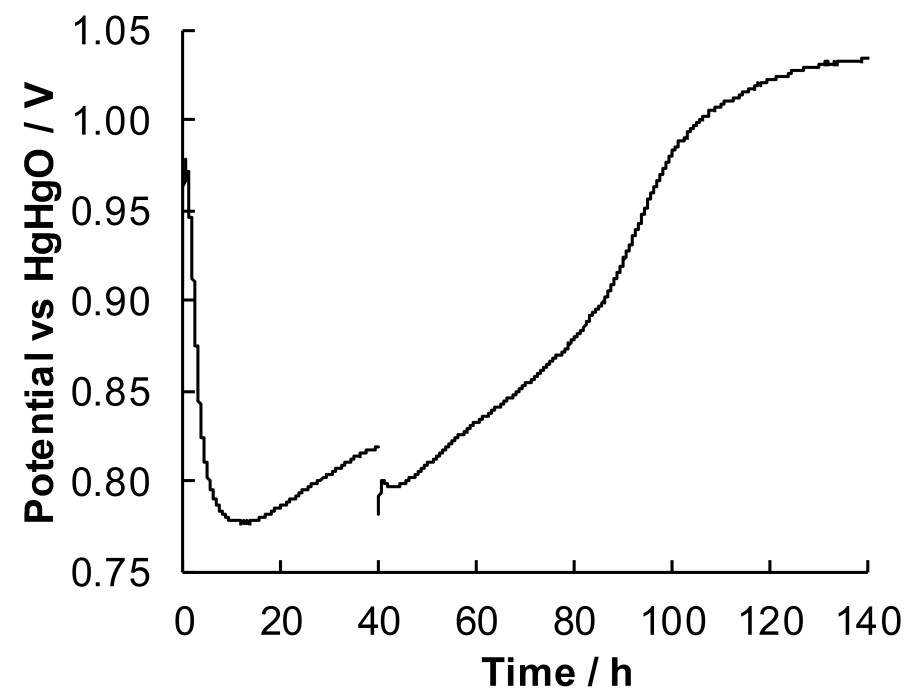

Figure 4: Galvanostatic oxygen evolution at $50 \mathrm{~mA} \mathrm{~cm}{ }^{-2}$ with a rejuvenation step at 40 hours. The potential was held at $0.5 \mathrm{~V}$ for 10 minutes during the rejuvenation step. 

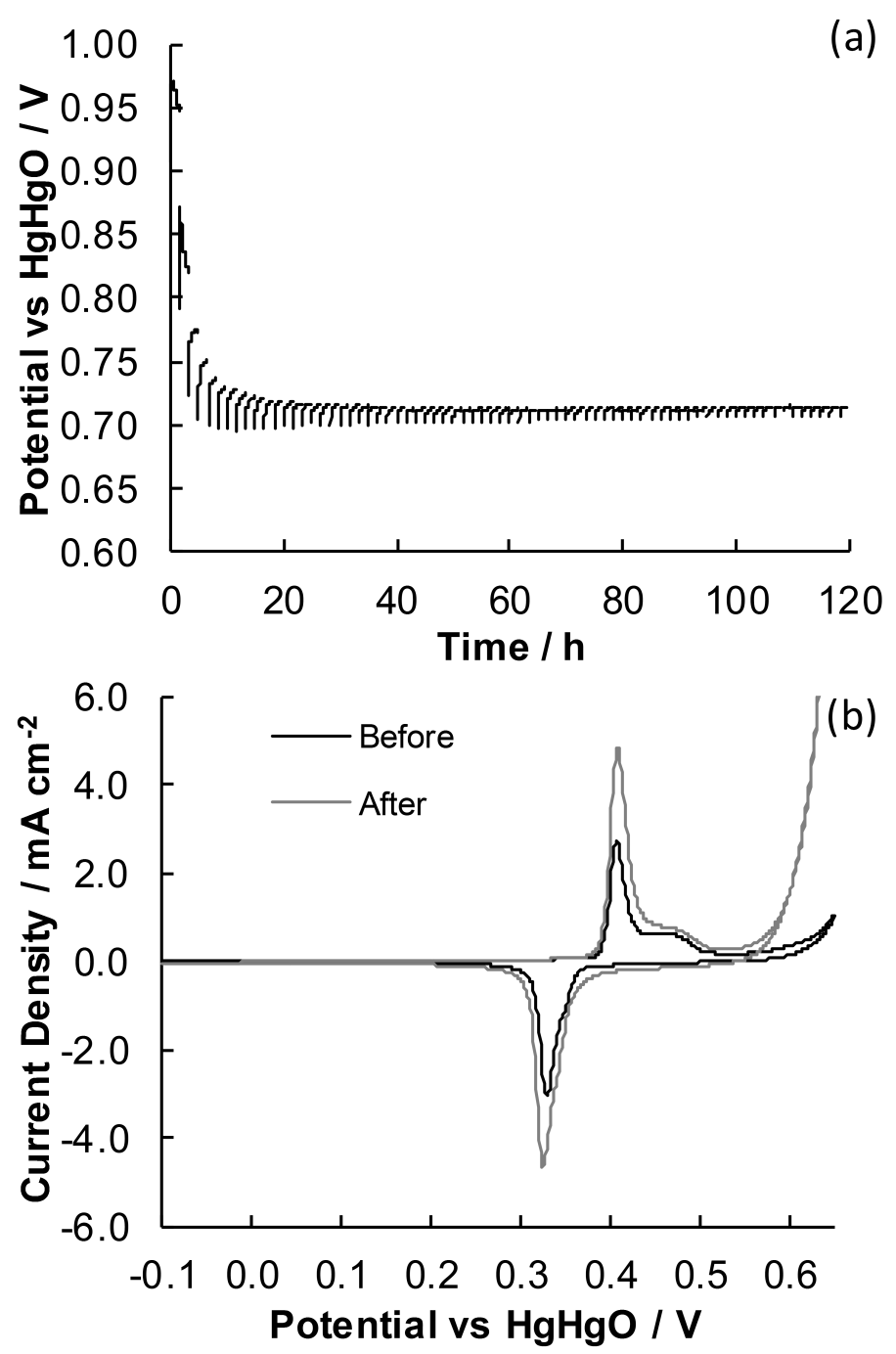

Figure 5: Galvanostatic oxygen evolution at $50 \mathrm{~mA} \mathrm{~cm}{ }^{-2}$ with rejuvenation steps down to $0.5 \mathrm{~V}$ for 10 minutes in every 100 minutes (a) and cyclic voltammograms at $50 \mathrm{mV} \mathrm{s}^{-1}$ between $-0.1 \mathrm{~V}$ and $0.65 \mathrm{~V}$ performed before and after oxygen evolution (b). 

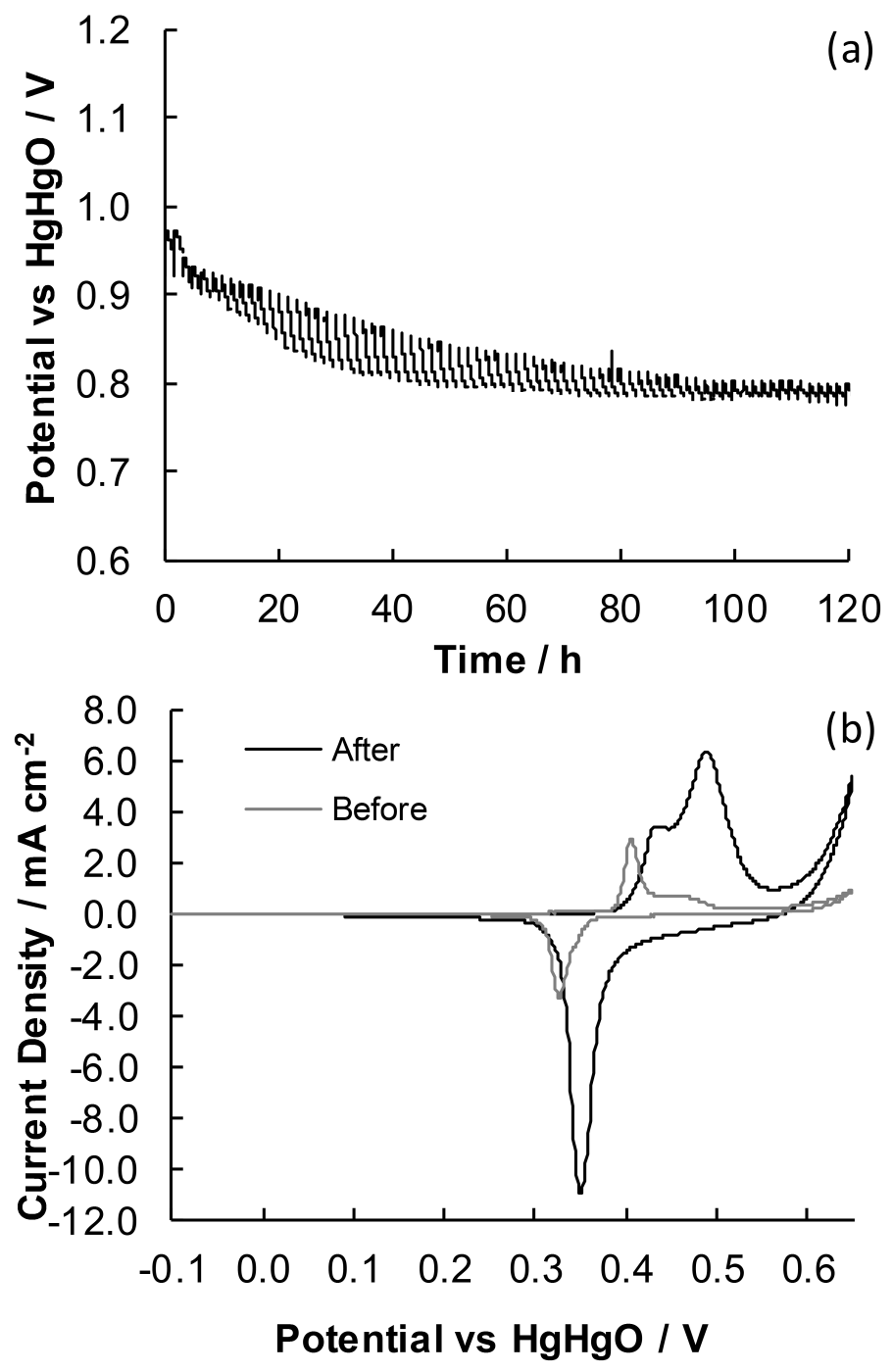

Figure 6: Galvanostatic oxygen evolution at $50 \mathrm{~mA} \mathrm{~cm}{ }^{-2}$ with rejuvenation steps down to $0.2 \mathrm{~V}$ for 10 minutes in every 100 minutes (a) and cyclic voltammograms performed at $50 \mathrm{mV} \mathrm{s}^{-1}$ between $-0.1 \mathrm{~V}$ and $0.65 \mathrm{~V}$ before and after oxygen evolution. 

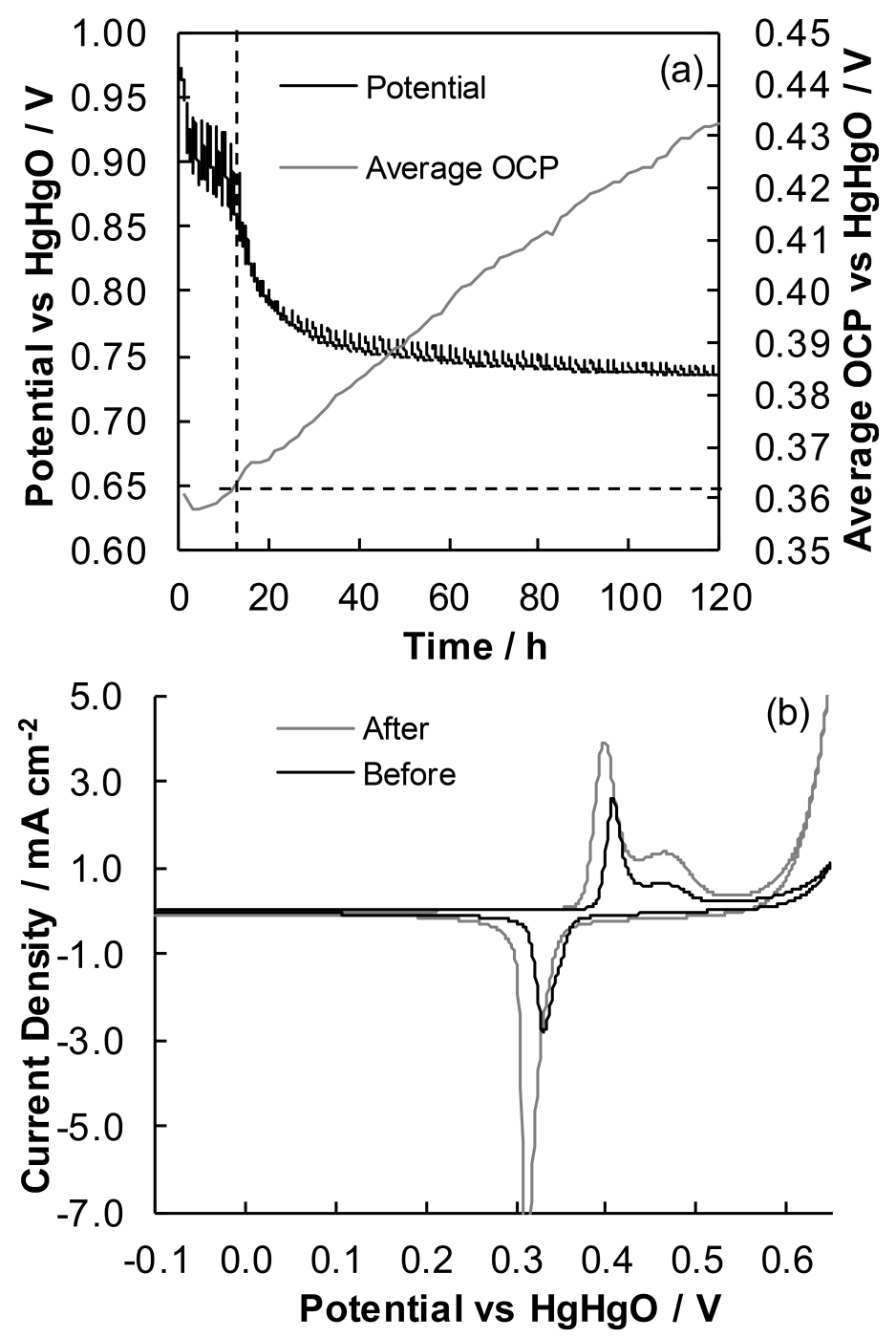

Figure 7: Galvanostatic oxygen evolution at $50 \mathrm{~mA} \mathrm{~cm}{ }^{-2}$ with rejuvenation steps at open circuit potential for 10 minutes in every 100 minutes (a) and cyclic voltammograms at $50 \mathrm{mV} \mathrm{s}^{-1}$ between $-0.1 \mathrm{~V}$ and $0.65 \mathrm{~V}$ performed before and after oxygen evolution at $50 \mathrm{mV} \mathrm{s}^{-1}$ between $-0.1 \mathrm{~V}$ and $0.65 \mathrm{~V}$ (b). The dashed lines in (a) indicate the time at which the OCP during the rejuvenation step exceeds $0.36 \mathrm{~V}$. 


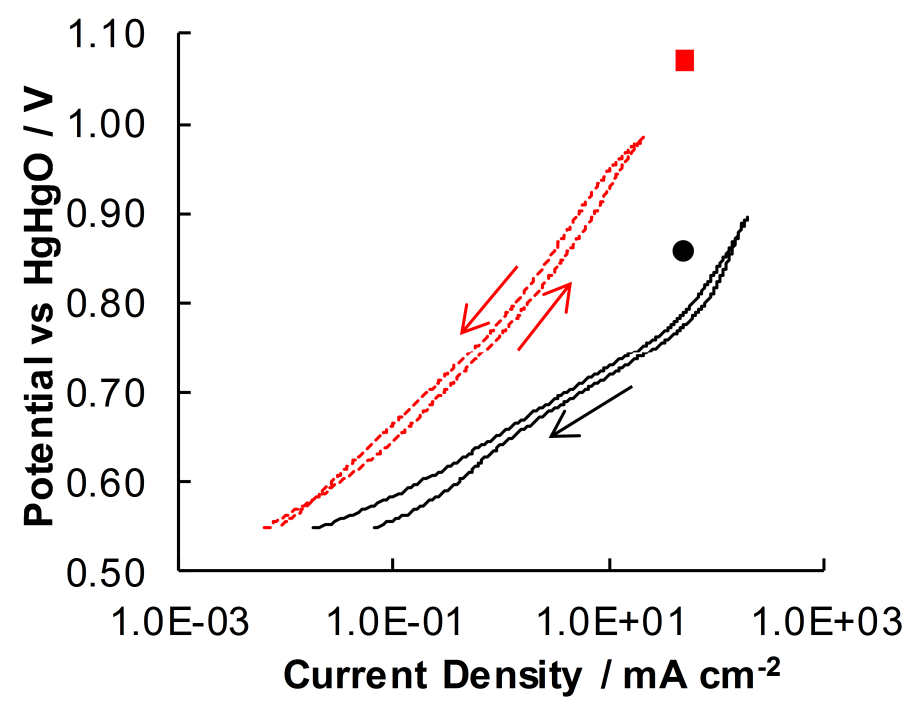

Figure 8: Slow linear scanning voltammetry from $0.55 \mathrm{~V}$ to $1.0 \mathrm{~V}$ and back after galvanostatic OER at $50 \mathrm{~mA} \mathrm{~cm}{ }^{-2}$ for 40 (dashed line) or 80 hours (solid line). The arrows indicate the direction of the potential sweep used to measure the polarisation data. The solid data points (circle - 40 hrs, square $80 \mathrm{hrs}$ ) give the potential at $50 \mathrm{~mA} \mathrm{~cm}^{-2}$ measured during the galvanostatic OER just prior to these polarisation measurements

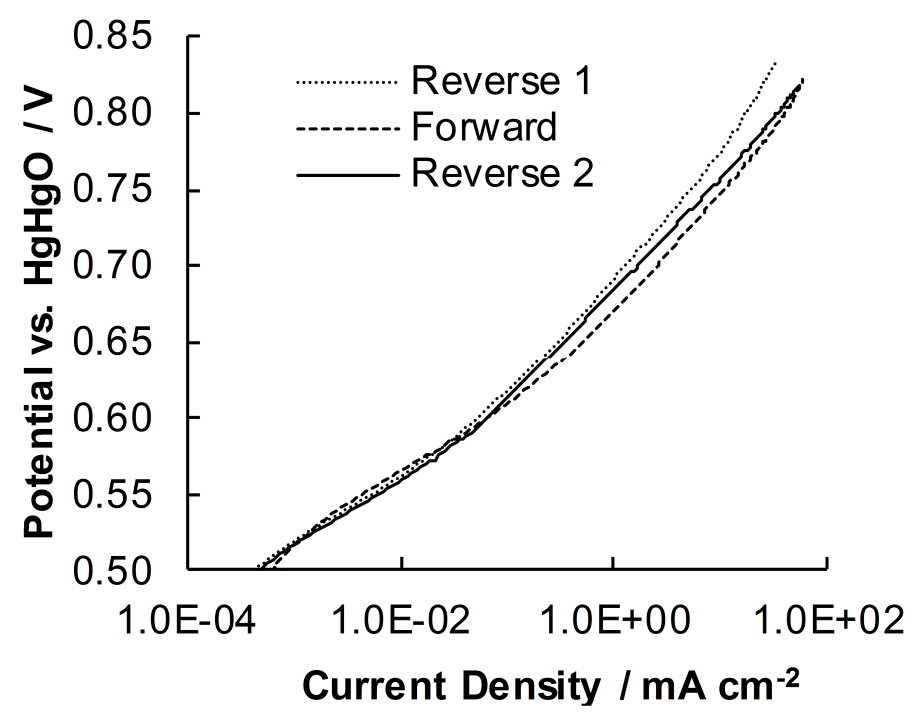

Figure 9: Slow linear scanning voltammetry between $0.5 \mathrm{~V}$ and $0.85 \mathrm{~V}$ (reverse, forward, reverse) after galvanostatic OER at $50 \mathrm{~mA} \mathrm{~cm}^{-2}$ for 45 hours. 